\title{
Metodologias ativas no cateterismo periférico venoso: desenvolvimento de habilidades com simulador de baixo custo
}

\author{
Active methodologies in peripheral venous catheterization: Skills development with a low-cost simulator \\ Metodologías activas en cateterismo venoso periférico: desarrollo de habilidades con simulador de bajo costo
}

\author{
Bruna Pedroso Canever ${ }^{1}$ (]) \\ Marina Silva Sanes ${ }^{2}$ (]) \\ Saionara Nunes de Oliveira ${ }^{3}$ (D) \\ Aline Lima Pestana Magalhães ${ }^{1}$ (D) \\ Marta Lenise do Prado 2,4 (1) \\ Diovane Ghignatti da Costa $^{1}$ (B)
}

1. Universidade Federal de Santa Catarina Departamento de Enfermagem. Florianópolis, SC, Brasil.

2. Universidade Federal de Santa Catarina, Programa de Pós-graduação em Enfermagem. Florianópolis, SC, Brasil.

3. Hospital Universitário Polydoro Ernani de São Thiago. Florianópolis, SC. Brazil

4. Universidade Federal do Amazonas, Programa de Pós-Graduação em Enfermagem. Manaus, AM. Brasil.

Autor correspondente: Bruna Pedroso Canever. E-mail: brunacanever@gmail.com.

Recebido em 22/04/2020.

Aprovado em 23/07/2020.

DOI:https://doi.org/10.1590/2177-9465-EAN-2020-0131

\section{RESUMO}

Objetivo: Conhecer a percepção de estudantes de enfermagem sobre a contribuição do uso do simulador de baixo custo no desenvolvimento de habilidades técnicas para o cateterismo periférico venoso. Método: Estudo descritivo de abordagem qualitativa, realizado em universidade pública do sul do Brasil com 25 estudantes de enfermagem. Os dados foram coletados em 2019, por meio de questionário acerca do cateterismo periférico venoso, desenvolvido com apoio de simulador de baixo custo. Para análise, seguiu-se a proposta operativa de Minayo. Resultados: A partir dos dados, surgiram duas categorias. 1) Desenvolvimento de habilidades para cateterismo periférico venoso: os estudantes apontaram que o uso do simulador possibilita compreender cada etapa do procedimento e identificar onde precisam aperfeiçoar a técnica, preparando-os para o contato com o paciente. 2) Dificuldades encontradas na utilização do simulador de baixo custo. Os estudantes destacaram a baixa fidelidade do simulador e a limitação do treinamento por simulação sem comunicação. Conclusões e implicações para a prática: Os estudantes percebem o simulador de baixo custo como uma relevante ferramenta para o desenvolvimento de habilidades do cateterismo periférico venoso, sugerem seu aperfeiçoamento para aumentar a fidelidade e a incorporação da comunicação no momento da punção para o maior realismo da experiência simulada.

Palavras-chave: Enfermagem; Ensino; Treinamento por Simulação; Cateterismo periférico; Estudantes de enfermagem.

\section{ABSTRACT}

Objective: To investigate nursing students' perception on the contribution of using a low-cost simulator in the development of technical skills for peripheral venous catheterization. Method: A qualitative and descriptive study carried out in a public university of southern Brazil. Data was collected from 25 nursing students in 2019 by means of a questionnaire about peripheral venous catheterization, developed with the support of a low-cost simulator. Data was analyzed using Minayo's operative proposal. Results: Two categories emerged from the data. 1) Skills development for peripheral venous catheterization: the students pointed out that the use of the simulator makes it possible to understand each stage of the procedure and to identify where they need to improve the technique, preparing them for contact with the patient. 2) Difficulties encountered in using the low-cost simulator. The students highlighted the low fidelity of the simulator and the limitation of the training by simulation without communication. Conclusions and implications for practice: The students perceive the low-cost simulator as a relevant tool for the development of venipuncture skills and they suggest its improvement to increase fidelity, as well as the incorporation of communication at the moment of puncture for greater realism of the simulated experience.

Keywords: Nursing; Teaching; Simulation Training; Peripheral Catheterization; Nursing Students.

\section{RESUMEN}

Objetivo: Conocer la percepción de los estudiantes de enfermería sobre el aporte de usar un simulador de bajo costo en e desarrollo de habilidades técnicas para cateterismo venoso periférico. Método: Estudio descriptivo con enfoque cualitativo, realizado con 25 estudiantes de enfermería en una universidad pública del sur de Brasil. La recopilación de datos se llevó a cabo en el año 2019, a través de un cuestionario sobre venopunción periférica, desarrollado con el apoyo de un simulador de bajo costo. Los datos se analizaron a través de la propuesta operativa de Minayo. Resultados: A partir de los datos surgieron dos categorías: 1) Desarrollo de habilidades para cateterismo venoso periférico: los estudiantes señalaron que utilizar e simulador permite comprender cada etapa del procedimiento e identificar dónde deben mejorar la técnica, lo que los prepara para el contacto con el paciente. 2) Dificultades encontradas al usar el simulador de bajo costo. Los estudiantes destacaron la baja fidelidad del simulador y la limitación para entrenar la técnica sin comunicación. Conclusiones e implicaciones para la práctica: los estudiantes perciben al simulador de bajo costo como una herramienta relevante para desarrollar habilidades en cateterismo venoso periférico, sugieren su perfeccionamiento para aumentar su fidelidad e incorporar la comunicación al momento de la punción para lograr mayor aproximación a la realidad en la experiencia simulada.

Palabras clave: Enfermería; Enseñanza; Entrenamiento Simulado; Cateterismo Periférico; Estudiantes de Enfermería. 


\section{INTRODUÇÃO}

A formação em enfermagem requer o desenvolvimento de competências específicas e, dentre estas, a competência técnica, a qual inclui o desenvolvimento de habilidades para a execução de atividades que subsidiarão a assistência em saúde nos serviços. Algumas dessas técnicas são de natureza invasiva e, por esta razão, os estudantes precisam aprendê-las em ambientes seguros para os envolvidos no processo de ensino-aprendizagem.

Essa condição extrapola a relação professor-estudante, considerando que a assistência é prestada nos serviços de saúde, incluindo-se como atores principais nesse processo os usuários dos serviços. ${ }^{1}$ Entre as técnicas invasivas que demandam estratégias inovadoras para aprendizagem, no intuito de promover o desenvolvimento de habilidades com segurança, tem-se o cateterismo periférico venoso ou a punção venosa periférica, cuja aprendizagem requer atenção e cuidados.

Com isso, torna-se premente a adoção de estratégias pedagógicas inovadoras, congruentes a cenários complexos de ensino-aprendizagem, conforme se identifica na área de conhecimento da enfermagem. As metodologias ativas vem subsidiar os docentes a atuarem em tais cenários, pois congregam concepções pedagógicas que estimulam o aprendizado de maneira crítica, reflexiva, ativado pelo indivíduo em formação, o qual aprende a aprender. ${ }^{2}$ Associar tais concepções ao processo de ensino-aprendizagem que envolve técnicas invasivas possibilita o desenvolvimento de competências de maneira segura, por meio do uso de estratégias voltadas ao ensino, como a simulação.

A simulação é um método de ensino e aprendizado baseado em evidências que tem sido usado na educação em enfermagem em países desenvolvidos há vários anos, ${ }^{3,4}$ e é uma prática crescente em todo o mundo. Além disso, a aprendizagem baseada em simulação tem sido apreciada pelos estudantes de enfermagem, pois apresenta experiências positivas como aumento do envolvimento na aprendizagem, diminuição dos níveis de ansiedade, satisfação e autoconfiança no aprendizado. ${ }^{5}$

Atualmente, observa-se o crescente uso de simuladores na educação em enfermagem, ${ }^{6}$ os quais são disponibilizados no mercado, por empresas especializadas, para atender a demanda de desenvolvimento de habilidades técnicas específicas de profissionais de saúde e de estudantes. Há inúmeros tipos de simuladores para o treinamento em saúde disponibilizados no mercado, desde partes para o treinamento de procedimentos únicos até simuladores de alta tecnologia. ${ }^{7}$ No entanto, o alto custo para adquirir e manter laboratórios equipados com simuladores de alta fidelidade tem sido apontado como um fator limitador de seu uso. ${ }^{8}$

O uso continuado desse recurso e a frequência necessária para atender os requerimentos de aprendizagem dos estudantes, impõem às escolas um alto custo financeiro, considerando que os equipamentos com maior fidelidade são muito caros e não são acessíveis a todos os educadores. ${ }^{9,10}$ Essa situação gera limitações no uso de equipamentos de simulação durante o processo formativo de estudantes de enfermagem.
Para que o aprendizado seja otimizado, o ambiente e o equipamento de simulação são projetados para refletir a realidade de um ambiente real de atendimento ao paciente. Esse grau de realismo ou de fidelidade varia entre baixa, média e alta, dependendo da complexidade da tarefa e do nível de conhecimento do estudante. ${ }^{11}$

A simulação de baixa fidelidade tem menos realismo e semelhança com o meio ambiente e/ou equipamentos do que é encontrado no ambiente clínico real. Esse tipo de simulação envolve o uso de manequins estáticos ou treinadores de tarefas, conhecidos como simuladores de baixo custo (SBC), projetados para ajudar o estudante a praticar uma habilidade psicomotora específica, como como punção venosa, sondagem nasogástrica, cateterismo vesical, entre outros. ${ }^{8,12}$ Além disso, por utilizar pouca tecnologia, os SBC envolvem manutenção simples e custos reduzidos, quando comparados aos dispositivos de alta fidelidade. ${ }^{13}$

Por esta razão, os SBC têm sido adotados em diferentes realidades no mundo e contribuído com o processo de ensinoaprendizagem de estudantes na área da saúde. ${ }^{8,14}$ É uma alternativa com viabilidade financeira, que amplia o oportunidade de treinamento, não apenas de estudantes, contribuindo também para o aperfeiçoamento técnico de trabalhadores, em ambiente seguro e controlado, promovendo uma aprendizagem segura, em especial, no desenvolvimento de habilidades psicomotoras.

Estudos indicam que as intervenções educacionais de enfermagem baseadas em simulação são eficazes, com efeitos particularmente grandes no domínio psicomotor. ${ }^{15,16}$ Todavia, há evidências de que o efeito do ensino de enfermagem baseado em simulação não indica ser proporcional ao nível de fidelidade. ${ }^{8}$ Ressalta-se, ainda, que a maioria das pesquisas em simulação no ensino de enfermagem envolvem simulação de alta fidelidade, existindo uma limitação em pesquisas com simulação de baixa fidelidade. ${ }^{17}$

No tocante ao cateterismo periférico venoso, um simulador de baixo custo pode ser construído pelos próprios professores e estudantes, com materiais simples e, muitas vezes, descartáveis. Consta na literatura um modelo de SBC desenvolvido no Brasil para tal finalidade, o qual foi avaliado por docentes como eficiente. ${ }^{18}$ Por tratar-se de tecnologia inovadora, a questão norteadora do presente estudo foi: Qual a contribuição do uso de um simulador de baixo custo para o desenvolvimento de habilidades técnicas dos estudantes para o cateterismo periférico venoso?

O objetivo deste estudo foi conhecer a percepção de estudantes de enfermagem sobre a contribuição do uso do simulador de baixo custo no desenvolvimento de habilidades técnicas para o cateterismo periférico venoso.

\section{MÉTODO}

Estudo descritivo, de abordagem qualitativa, desenvolvido em uma universidade pública do sul do Brasil. O cenário de pesquisa foi o laboratório de práticas de enfermagem, cujas 
atividades teórico-práticas incluem a primeira disciplina em que os estudantes entram em contato com técnicas que subsidiam a assistência de enfermagem. A ementa da disciplina contempla, entre diversos outros assuntos, o desenvolvimento de habilidades técnicas do cateterismo periférico venoso. Em sua programação estão previstos três encontros, um teórico e dois práticos, para abordar o conteúdo de cuidados de enfermagem no cateterismo periférico venoso e fluidoterapia, antes dos estudantes irem ao cenário real, o hospital.

Nas atividades práticas, os estudantes utilizaram um SBC para o cateterismo periférico venoso. Trata-se de um protótipo que reproduz parte de um membro superior em que é possível visualizar e palpar uma veia, colocar o garrote, puncionar, visualizar o refluxo do sangue, instalar a fluidoterapia ou administrar medicamentos em bolus, ou ainda coletar sangue, conforme pode ser visto na Figura 1. A construção ocorreu a partir de um modelo apresentado no Congresso Latino-americano de Simulação realizado no Chile e validado por docentes de uma Instituição Federal de Ensino Superior. ${ }^{18}$

Antes de iniciar a aula prática, os docentes apresentaram o simulador de baixo custo aos estudantes, explicando como funcionava e demonstrando o passo a passo do cateterismo

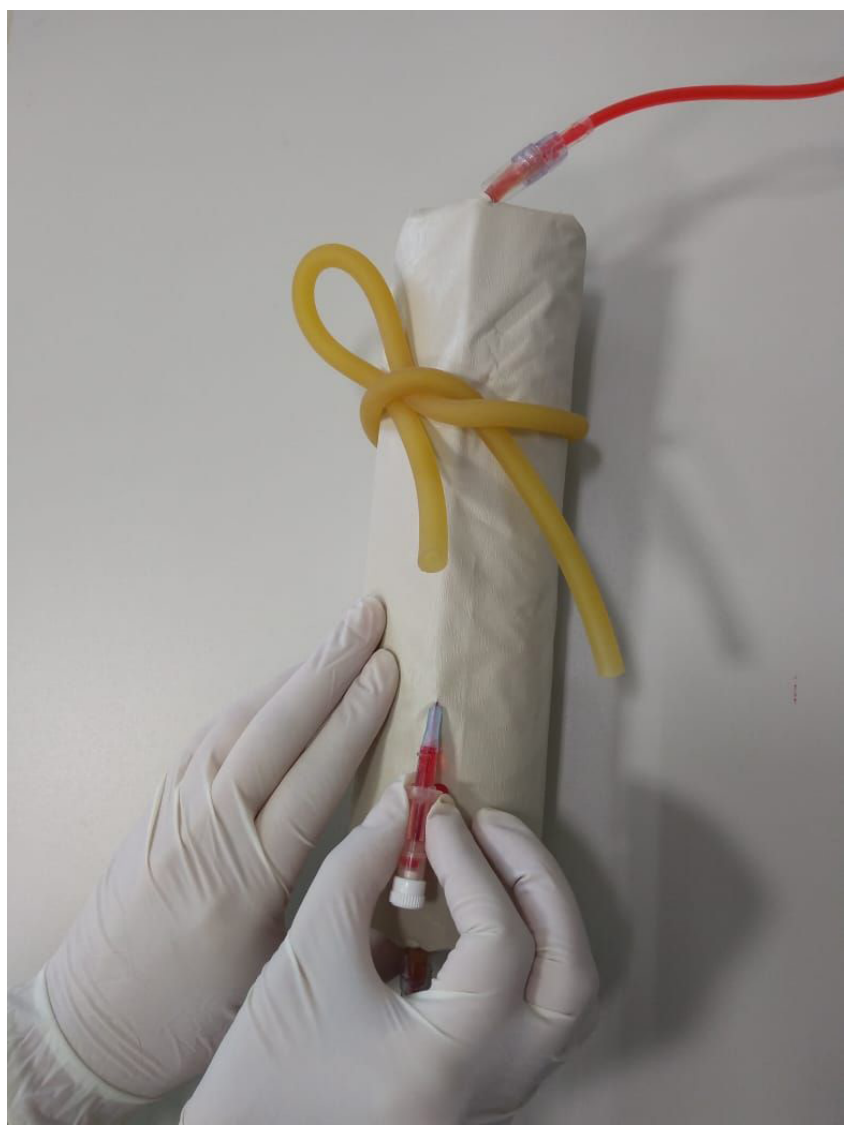

Figura 1. Simulador de Baixo Custo. Florianópolis-SC, 2019. Fonte: Arquivo pessoal. periférico venoso e instalação da fluidoterapia. O tempo utilizado para este diálogo foi de 50 minutos.

Logo após esse momento, os alunos foram divididos em três grupos para a realização da prática com o simulador. Cada grupo foi acompanhado por um professor, em salas distintas do laboratório e o tempo de duração da prática foi de 2 horas e 50 minutos. Além do simulador de baixo custo, foram utilizados os seguintes materiais para a prática: garrote, cateter sobre agulha, agulha, seringa, cateter agulhado, tubos de coleta a vácuo, equipo, soro fisiológico, algodão, álcool, luvas de procedimento. Cada estudante teve a oportunidade de realizar três procedimentos (coleta de sangue, punção cateter agulhado e punção com cateter sobre agulha com instalação de fluidoterapia). O professor acompanhou o desenvolvimento da prática simulada observando, orientando e dando feedback, sempre que necessário. Em um momento final, os estudantes ainda puderam retomar dúvidas acerca dos procedimentos executados.

O estudo contou com 25 participantes matriculados na disciplina pesquisada no semestre de coleta, constituindo uma amostra por conveniência. A saturação de dados norteou a decisão que se alcançou o suficiente para responder ao objetivo da pesquisa. ${ }^{19}$

Considerou-se como critério de inclusão ser estudante do curso de graduação em enfermagem matriculado na disciplina responsável por desenvolver as competências relacionadas ao cateterismo periférico venoso e fluidoterapia. O critério de exclusão adotado consistiu em estar ausente em pelo menos um dos três encontros para desenvolvimento das atividades sobre a temática. Dez estudantes foram excluídos do estudo.

A coleta de dados foi conduzida pela pesquisadora principal, no primeiro semestre de 2019, nos meses de abril e maio, após a realização do terceiro encontro, o qual finalizou as atividades teórico-práticas em laboratório.

Os dados foram coletados por meio de questionário composto por perguntas abertas referentes ao uso do simulador de baixo custo, que versavam acerca de: percepção em relação ao uso do simulador de baixo custo para o desenvolvimento da técnica do cateterismo periférico venoso; etapas do procedimento cateterismo periférico venoso e o uso do simulador; vantagens e desvantagens no uso de simulador de baixo custo; sugestões de aperfeiçoamento.

Após transcrição literal, as informações foram analisadas segundo a proposta operativa de Minayo ${ }^{20}$, composta por dois momentos operacionais. $\mathrm{O}$ primeiro momento mapeia as determinações fundamentais do estudo, e o outro momento, conhecido como interpretativo, consiste na representação do encontro com os fatos empíricos. O segundo momento operativo apresenta duas etapas: a ordenação e a classificação dos dados, que abrange a leitura horizontal e exaustiva dos textos, leitura transversal, análise final e construção do relatório com a apresentação dos resultados. O processamento das informações resultou em duas categorias. 
Quanto aos aspectos éticos, a pesquisa foi aprovada sob parecer CAAE- 82218418.0.0000.0121, contando-se com a anuência dos participantes mediante assinatura individual do termo de consentimento livre e esclarecido. Para preservar o anonimato dos participantes do estudo, utilizou-se a identificação alfanumérica $E$ (estudante), seguida de números de 1 a 25 , correspondendo ao número de participantes, os quais foram ordenados sequencialmente conforme ordem de entrega do questionário.

\section{RESULTADOS}

As percepções dos participantes em relação ao uso do simulador de baixo custo para o treinamento de cateterismo periférico venoso apontaram duas categorias: desenvolvimento de habilidades para o cateterismo periférico venoso e dificuldades encontradas na utilização do simulador de baixo custo.

\section{Desenvolvimento de habilidades para o cateterismo periférico venoso}

O desenvolvimento de habilidades para o cateterismo periférico venoso na formação em enfermagem requer a integração de conhecimentos teóricos, práticos e atitudinais, os quais se apresentam de maneira concomitante durante sua execução. Trata-se de um dos primeiros procedimentos invasivos que os estudantes aprendem a realizar, o que mobiliza expectativas e sentimentos para tal aprendizagem.

A utilização do simulador de baixo custo para o desenvolvimento de habilidades técnicas do cateterismo periférico venoso demonstrou-se como um recurso relevante para a aprendizagem dos estudantes, pois permitiu a identificação de etapas do treinamento por simulação que mais contribuíram para o processo de aprendizagem, segundo as percepções de cada participante. Os registros a seguir apresentam as diferentes etapas da técnica que foram destacadas pelos estudantes:

\section{A inserção do equipo ao cateter, pois mostrou a agilidade que necessito (E7). \\ Eu tinha muita dificuldade de colocar o garrote e foi neste simulador que eu aprendi (E22). \\ A palpação para achar o vaso, pois foi a etapa mais similar (E12). \\ A punção, pela resistência da agulha; simula muito bem a pressão oferecida ao vaso (E15).}

Outro ponto realçado pelos estudantes foi que o simulador possibilita aprendizagem a partir da explicação do professor e da identificação dos próprios erros, permitindo refletir sobre a etapa da técnica que ainda necessitava de aprimoramento:

\section{A prática, pois ali erramos e pensamos a respeito (E10).}

A prática mesmo, e a demonstração do professor ajudou muito, explicar o passo a passo (E11).
Antes não tinha noção de como deveria ser feito, com o equipamento pude perceber qual era o maior desafio e melhorar (E13).

Os estudantes destacaram como vantagens no uso do simulador, a possibilidade da execução do procedimento numa situação controlada antes de realizá-lo no paciente, permitindo a experiência prévia, bem como a compreensão das etapas a serem realizadas:

Temos uma experiência boa antes de treinar no campo de prática (E18)

As vantagens são baseadas no princípio de que o simulador auxilia na visão real dos fatos a serem realizados (E15).

Ao relacionar a experiência educativa à prática no cenário real, os estudantes identificaram o treinamento por simulação e o espaço como ambientes seguros para a aprendizagem, além da demonstração de um compromisso ético com o ser humano ao refletirem sobre não ser possível fazer este o treinamento por simulação numa pessoa que não necessita de tal intervenção, aqui identificada como um colega.

Não permite erros maiores e consequências [a prática no cenário real]. Liberdade essa que seria impossível num colega. Permite-nos adquirir as habilidades sem causar consequência (E9).

É de grande valia o aprendizado com o simulador, pois ele é simples, prático e nos ajuda a simular a punção com o refluxo de sangue (E2).

\section{Dificuldades encontradas na utilização do simulador de baixo custo}

Os estudantes apontaram como dificuldades a presença de orifícios e marcas que ficam no simulador após várias tentativas de punção, pois indicam o local de punção de modo visual. Outro aspecto refere-se à distância da técnica, quando se compara a uma situação real de cateterismo periférico venoso, uma vez que não se assemelha a um membro superior. Além disso, o dispositivo apresenta apenas uma veia, condição que direciona sua localização e que pode não representar as condições reais para a realização da técnica, como a variação das condições clínicas dos pacientes. Os registros a seguir ilustram essas percepções:

[...] ficam furos depois que inserimos a agulha, ele poderia ser um pouco melhor nesse sentido (E2).

[...] apenas um vaso, torna mais óbvio a localização, principalmente com as marcas deixadas pelas agulhas a cada tentativa (E10).

O simulador poderia ser mais parecido com um membro e não acoplado a um corpo, se distanciando da realidade (E5). 
Para o aprimoramento do simulador, os estudantes sugeriram algumas modificações para deixá-lo mais semelhante com a anatomia de um membro, entre outras, a conveniência de a superfície que recobre o simulador ser parecida com a pele e dispor de maior quantidade de veias com diferentes diâmetros.

\section{[...] melhorando a superfície ficando mais parecido com a pele (E9).}

Aumento do peso e rigidez do braço e aumentar o número de veias com diâmetros diferentes seria muito interessante (E4).

[...] precisaria ser um pouco mais pesada e mais fiel a anatomia (E13).

Ainda sobre as fragilidades encontradas no uso do simulador, os estudantes destacaram a dificuldade de ter a noção exata da realidade, bem como a impossibilidade do treinamento de competências relacionais, como a comunicação, pois tratava-se de um membro.

\section{[...] que não nos deu total/maior noção da realidade do procedimento. Como tensão do tecido, etc (E24).}

Não é muito fiel ao real, não tivemos a possibilidade de treinar a comunicação (E18).

\section{DISCUSSÃO}

Um dos elementos fundamentais na graduação de enfermagem é o desenvolvimento de competências a partir do conhecimento, habilidades e atitudes, as quais são aprimoradas ao longo do curso pelos estudantes. Sabe-se que existe uma expectativa por parte dos estudantes de enfermagem referente às técnicas mais específicas como sondagens, aferição de pressão arterial, curativo e cateterismo periférico venoso, sendo este último foco deste estudo.

Neste contexto, o ensino competente representa o saber e o saber-fazer articulados às dimensões científicas, técnicas, éticas, políticas, relacionais e estéticas da formação. Para que o estudante alcance a competência técnica, faz-se necessário mobilizar um conjunto de elementos que permitam organizar o raciocínio lógico de cada etapa do procedimento, necessitando articular conhecimentos, habilidades e atitudes durante sua execução. ${ }^{21}$

No caso da disciplina em que se utilizou o SBC, promoveu-se aproximação aos conhecimentos técnico-científicos, subsidiando os estudantes no desenvolvimento da destreza, mediante o exercício de etapa por etapa do procedimento, em condições controladas e seguras. Ou seja, tal habilidade foi desenvolvida em momento anterior ao cenário de práticas de campo de estágio, entendendo-se que o SBC minimiza a ansiedade e expectativa dos estudantes, bem como os riscos de danos desnecessários associados à assistência aos pacientes, porque é um momento sem a presença real do paciente..$^{22}$
O uso do SBC permite, ainda, dentro do desenvolvimento pleno/total da habilidade, que o estudante possa dividir a técnica em outras habilidades menores como fazer antissepsia, garrotear, puncionar, coletar sangue ou instalar fluidoterapia. Ou seja, é a reunião de habilidades menos complexas, orquestradas dinamicamente numa ordem adequada, que dá condições de um aprendiz conhecer suas facilidades na realização da técnica, bem como identificar seus desafios..$^{23} \mathrm{E}$ isso, na construção do conhecimento sobre cateterismo periférico venoso, acontece gradativamente, haja vista se tratar de algo processual e sistematizado.

Nesse sentido, a importância da relação de ensinoaprendizagem entre professor e estudante reside em identificar as etapas bem desenvolvidas e aquelas que necessitam de aprimoramento. No SBC, há possibilidade de desenvolvimento de cada etapa da técnica, permitindo ao estudante ter consciência, com a mediação do professor, dos aspectos em que precisa melhorar. Além disso, é possível parar em qualquer momento, revisar o procedimento e retornar ao processo sem acarretar prejuízos e/ou riscos. ${ }^{24,25}$

A possibilidade de pormenorizar a realização da técnica com o uso do simulador tem relevância pedagógica na perspectiva de construção processual do conhecimento. Essa construção ocorre mediante a integração inicial dos conhecimentos técnicocientíficos, os quais serão associados, em momento posterior, às atitudes necessárias para que o cuidado de enfermagem configure em uma ação competente e segura.

$O$ ato de conscientizar-se permite ao estudante o desenvolvimento da reflexão, aumentando a compreensão das suas necessidades no processo educativo. O processo de conscientização possibilita o encontro com um mundo subjetivo, que o estudante conhece à medida que se conhece. Nesse encontro, ocorre um movimento de transcendência da visão ingênua até a apreensão da realidade, condição em que o indivíduo assume uma postura crítica sobre o próprio conhecimento. ${ }^{26}$

O desenvolvimento de habilidades técnicas, a partir do uso do SBC, possibilita uma aprendizagem experiencial do estudante em um ambiente seguro, controlado e mais próximo do real. Dessa forma, reitera-se que a simulação como estratégia de ensino permite aos estudantes vivenciar de forma ativa um contexto específico, garantindo a sua prática com autonomia e evitando possíveis eventos adversos aos pacientes. ${ }^{27}$ Construir conhecimentos com o uso de metodologias ativas permite 0 protagonismo do estudante de enfermagem, especialmente porque situações geradoras de ansiedade e estresse podem aparecer e ser discutidas no contexto pedagógico. ${ }^{28}$

OSBC permite que os estudantes identifiquem erros durante o desenvolvimento de habilidades e referem à importância desse momento para o processo de aprendizagem. Assim, as atividades de simulação possibilitam aos estudantes pensar no seu processo educativo como aprendizes, tomando consciência e refletindo sobre a sua prática, ressignificando seu modo de pensar e agir, aperfeiçoando práticas e técnicas a serem utilizadas, de forma mais efetiva, com o paciente no ambiente de saúde. ${ }^{29} \mathrm{Com}$ isso, 
a articulação de competências que os estudantes empregam durante estratégias ativas de ensino, como a simulação, ultrapassam o nível técnico do procedimento, gerando reflexão e uma consequente transformação do aprendizado..$^{30}$

Estudo recente sobre a relevância de metodologias ativas, nos cursos de enfermagem, aponta que uma formação orientada por valores é capaz de transcender as competências de futuros profissionais de enfermagem. ${ }^{31}$ Considera-se que tais valores, no que concerne a esta pesquisa, são expressos na responsabilidade de desenvolver competências em ambientes seguros. Isso, quando se compara as condições em relação ao treinamento por simulação no SBC e diretamente em seres humanos, tanto entre colegas estudantes como em pacientes durante práticas em campos de estágio. O processo de aprendizagem com SBC em ambiente favorável, guiado pelo docente, gera a possibilidade de errar e de aprender com o erro, tanto sobre a técnica em si, como sobre as implicações que dela decorrem, caso fosse realizada em ambientes não simulados, diretamente em seres humanos. Essa condição tece uma rede de reflexões, a qual envolve fatores muito além do desenvolvimento da habilidade técnica em si, haja vista a complexidade da valoração humana e da rede de relações presentes na prestação de serviços de saúde.

Ao utilizar o SBC, com atenção para a experiência dos estudantes de enfermagem, é possível problematizar como um treinamento pode trazer questões relevantes para a formação profissional na enfermagem. $O$ uso do SBC no contexto de metodologias ativas permite que noções de segurança e de responsabilidade possam ser trabalhadas, demonstrando que o uso destes recursos está orientado pela ética. ${ }^{32}$

Em diferentes âmbitos, pesquisas comprovam que utilizar a simulação como estratégia de ensino é eficiente no sentido de melhorar a obtenção de conhecimentos, o desempenho e a segurança dos estudantes e, também, de profissionais da saúde em diversos procedimentos assistenciais. ${ }^{28,33-35}$ No entanto, a escolha por cenários complexos pode não atender às necessidades do que se pretende desenvolver. A preparação docente e o planejamento reúnem duas atividades essenciais para que a intencionalidade pedagógica se expresse na oferta de atividades educativas que realmente respondam aos objetivos de aprendizagem. Para habilidades técnicas, a centralidade está no treinamento repetitivo de determinada ação e/ou sequência, seguido de feedback. Nesse sentido, verificam-se vantagens para os cursos de enfermagem com a utilização de SBC, pois permite o desenvolvimento de habilidades técnicas por meio da realização repetitiva dos procedimentos, sempre que necessário, para assegurar o domínio pelo estudante, sem acarretar aumento de custos significativos.

A possibilidade de treinar exaustivamente procedimentos invasivos em simuladores permite experimentar a sensação do real. Procedimentos como sondagens, cateterismos, punções, auscultas, manobras de ressuscitação, comunicação e trabalho em equipe são algumas das possibilidades que a simulação e os simuladores oferecem. ${ }^{36}$ Estudo realizado na Suécia, com estudantes de enfermagem no treinamento de venopunção identificou correlação entre melhores desempenhos e autotreinamento, o que corrobora a potencialidade do uso de SBC na formação de enfermeiros por permitir inúmeras tentativas. ${ }^{23}$

O uso de SBC, no entanto também apresenta fragilidades. A baixa fidelidade do equipamento limita a percepção de ambiente real, em especial, a condição de sua execução em pacientes reais. Todavia, a segurança decorrente de seu uso, tanto dos estudantes quanto dos pacientes, na aprendizagem de técnicas invasivas relativiza as limitações identificadas no estudo. Um ambiente controlado e realista permite ao estudante vivenciar espaços e treinar técnicas e/ou procedimentos, dando-lhe oportunidade de errar e aprender com os erros, sem que esses erros tragam prejuízos à saúde de indivíduos. ${ }^{36}$

Apesar de se constatar a necessidade de melhorias, como aparência e fidedignidade do dispositivo apontadas na categoria sobre as dificuldades encontradas na utilização do SBC, de modo geral, sua utilização na presente pesquisa contribuiu para a aprendizagem dos estudantes, possibilitando avanços no processo de ensino de aprendizagem. Resultados semelhantes no ensino de enfermagem foram encontrados em outros estudos, em cenários diferentes, e com diferentes tipos de dispositivos, tais como: no cuidado da criança e da família; ${ }^{24}$ na saúde mental ${ }^{37}$ no cuidado a pessoas idosas. ${ }^{38}$

Outro ponto descrito pelos estudantes, categorizado como dificuldades, refere-se ao treinamento de competências relacionais, como a comunicação, a qual foi insuficiente por tratar-se de simulação com somente um membro, especialmente por sua aparência diferir sobremaneira de uma situação real. Resultados de pesquisas destacam a comunicação como uma habilidade possível de ser desenvolvida e aprimorada por meio de simulações, quando o simulador interage com o estudante, incluindo reações e comunicação não verbal, condições que o SBC não oferece. ${ }^{37,39}$

Diante dos resultados, considera-se que é conveniente proporcionar formação profissional ancorada em processos participativos e problematizadores, com o uso de metodologias ativas em ambiente de simulação, tanto sob o ponto de vista pedagógico, como de viabilidade financeira. Por outra parte, destaca-se que promovem reflexão sobre o processo de ensinoaprendizagem, tal qual se constatou nesta pesquisa com uso de SBC para desenvolvimento de habilidades do cateterismo periférico venoso. Tal processo, de característica dinâmica, alcança tanto o nível individual, de autoconhecimento, como o coletivo, por meio de observações de si e do outro na realização da técnica, mediante interações entre professores e estudantes, além de estudantes entre si.

\section{CONCLUSÕES E IMPLICAÇÕES PARA A PRÁTICA}

A técnica do cateterismo periférico venoso é um procedimento que oferece riscos e gera ansiedade tanto em quem executa quanto no paciente. Durante a formação, os estudantes de enfermagem precisam ter habilidade e segurança antes de 
executá-la em cenários reais, na assistência direta aos pacientes. Dessa forma, resgatando o objetivo deste estudo, evidencia-se que a utilização de simuladores de baixo custo foi considerada pelos estudantes uma experiência positiva que, além de contribuir com o desenvolvimento da habilidade técnica do cateterismo periférico venoso, proporcionou aprendizado sem risco de causar dano desnecessário.

Aponta-se como limitação deste estudo a fidedignidade do SBC, que apesar de apresentar avanços no envolvimento dos estudantes na realização da técnica, no treinamento exaustivo, tal característica foi apresentada como elemento central de desvantagem. No entanto, os estudantes apontaram sugestões de melhoria para o SBC, com as quais será possível aperfeiçoálo para que se torne mais próximo da realidade, no sentido de pensar aproximações em relação à pele, à aparência dos vasos, à sensibilidade na punção da veia, dentre outros aspectos. Ainda são necessários estudos experimentais com o intuito de comparar os efeitos do uso ou não do SBC no desenvolvimento de competências para o cateterismo periférico venoso.

O estudo apresenta contribuições para o processo de ensino e aprendizagem, pois demonstrou que o desenvolvimento de habilidades técnicas na formação em enfermagem pode incorporar recursos, técnicas e simuladores de baixa complexidade. Destaca-se, que tal medida deve se mostrar acompanhada de intencionalidade pedagógica em todas as etapas do processo. Cursos de graduação em enfermagem podem se utilizar desta experiência para criar possibilidades condizentes com sua realidade e à realidade dos estudantes.

Noutra perspectiva, mesmo que o cateterismo periférico venoso esteja substancialmente relacionada ao desenvolvimento de habilidade técnica, há que se dar luzes às noções de ética, responsabilidade e problematização, como partes de igual relevância para o processo educativo. Tal amplitude de reflexão faz-se necessária, haja vista a complexidade que envolve melhorar a situação de saúde de alguém que necessita de cuidados de enfermagem, a exemplo de um cateterismo periférico venoso, a qual deverá estar amparada na ética, no respeito ao ser humano e na segurança do paciente. Desse modo, o ensinar-aprender competências acerca do cateterismo periférico venoso também deve aproximar-se desses pressupostos.

\section{CONTRIBUIÇÕES DOS AUTORES}

Desenho do estudo. Bruna Pedroso Canever, Marina Silva Sanes, Saionara Nunes de Oliveira.

Coleta ou produção dos dados. Bruna Pedroso Canever, Marina Silva Sanes, Saionara Nunes de Oliveira, Aline Lima Pestana Magalhães.

Análise de dados e interpretação dos resultados. Bruna Pedroso Canever, Marina Silva Sanes, Saionara Nunes de Oliveira, Aline Lima Pestana Magalhães.

Redação e revisão crítica do manuscrito. Aprovação da versão final do artigo. Responsabilidade por todos os aspectos do conteúdo e a integridade do artigo publicado. Bruna Pedroso Canever, Marina Silva Sanes, Saionara Nunes de Oliveira, Aline
Lima Pestana Magalhães, Marta Lenise do Prado, Diovane Ghignatti da Costa.

\section{EDITOR ASSOCIADO}

Cândida Caniçali Primo.

\section{REFERÊNCIAS}

1. Gomes BKG, Martins AG, Lopes JR, Barbosa HA, Souto DF, Macie APF, et al. Conhecimento da equipe de enfermagem sobre inserção, manutenção e complicações relacionados ao cateter venoso periférico. REAS/EJCH. 12(8):e3408. https://doi.org/10.25248/reas.e3408.2020.

2. Macedo KDS, Acosta BS, Silva EB, Souza NS, Beck CLC, Silva KKD. Active learning methodologies: possible paths to innovation in health teaching. Esc Anna Nery. 2018:22(3):e20170435. http://dx.doi. org/10.1590/2177-9465-ean-2017-0435

3. Hayden JK. The NCSBN national simulation study: a longitudinal, randomized, controlled study replacing clinical hours with simulation in prelicensure nursing education. J Nurs Regul. 2014;5(2):S3-S40. http://dx.doi.org/10.1016/S2155-8256(15)30062-4.

4. Cant RP, Cooper SJ. Use of simulation-based learning in undergraduate nurse education: an umbrella systematic review. Nurse Educ Today. 2017;49(1):63-71. http://dx.doi.org/10.1016/j.nedt.2016.11.015 PMid:27902949.

5. OmerT. Nursing students' perceptions of satisfaction and self-confidence with clinical simulation experience. J Educ Pract. 2016;7(5):131-8.

6. Oliveira SNO, Prado ML, Kempfer SS. Utilização da simulação no ensino da enfermagem: revisão integrativa. REME - Rev Min Enferm. 2014;18(2):487-504. http://www.dx.doi.org/10.5935/1415-2762.20140036.

7. Waxman KT, Bowler F, Forneris SG, Kardong-Edgren S, Rizzolo MA Simulation as a Nursing Education Disrupter. Nurs Adm Q. 2019;43(4):3005. http://dx.doi.org/10.1097/NAQ.0000000000000369. PMid:31479049.

8. Muckler VC, Kampo S, Morgan B. Creation of a low-cost simulated trachea for deliberate practice of cricothyrotomy and retrograde wire use. AANA J. 2017;85(4):271-5. PMid:31566546.

9. Parry M, Fey MK. Simulation in advanced practice nursing. Clin Simul Nurs. 2019;26:1-2. http://dx.doi.org/10.1016/j.ecns.2018.11.004.

10. Kim J, Park J, Shin S. Effectiveness of simulation-based nursing education depending on fidelity: a meta-analysis. BMC Med Educ. 2016;16(1):152. http://dx.doi.org/10.1186/s12909-016-0672-7. PMid:27215280.

11. Sawyer T, White M, Zaveri P, Chang T, Ades A, French H et al. Learn, see practice, prove, do, maintain: an evidence-based pedagogical framework for procedural skill training in medicine. Acad Med. 2015;90(8):1025-33. http://dx.doi.org/10.1097/ACM.0000000000000734. PMid:25881645.

12. Ravik M, Havnes A, Bjørk IT. Exploring nursing students' transfer of peripheral venous cannulation from skills centre to the clinical setting J Nurs Educ Pract. 2015;5(3):59-70. http://dx.doi.org/10.5430/jnep. v5n3p59.

13. Mücke U, Grigull L, Sänger B, Berndt LP, Wittenbecher H, Schwarzbard $\mathrm{C}$ et al. Introducing low-cost simulation equipment for simulation-based team training. Clin Simul Nurs. 2019;38:18-22. http://dx.doi.org/10.1016/j. ecns.2019.09.001.

14. Lichtenberger JP, Tatum PS, Gada S, Wyn M, Ho VB, Liacouras P. Using 3D printing (Additive Manufacturing) to produce low-cost simulation models for medical training. Mil Med. 2018;183(Suppl 1):73-7. http:// dx.doi.org/10.1093/milmed/usx142. PMid:29635555.

15. Cant RB, Cooper SJ. Use of simulation-based learning in undergraduate nurse education: an umbrella systematic review. Nurse Educ Today. 2019;49:63-71. http://dx.doi.org/10.1016/j.nedt.2016.11.015 PMid:27902949.

16. Silva JP, Pereira Jr GA, Meska MHG, Mazzo A. Construção e validação de simulador de baixo custo para capacitação de pacientes com diabetes mellitus e/ou de seus cuidadores na aplicação de insulina. Esc Anna Nery. 2018;22(3):e20170387. http://dx.doi.org/10.1590/2177-9465ean-2017-0387. 
17. Ross JG. Simulation and psychomotor skill acquisition: a review of the literature. Clin Simul Nurs. 2012;8(9):429-35. http://dx.doi.org/10.1016/j. ecns.2011.04.004.

18. Oliveira SN, Canever BP, Silveira NIR, Fernandes SR, Martini JG, Lino MM. Simulador de baixo custo para punção venosa periférica: da confecção à avaliação. Rev enferm UERJ. 2019;27:e45584. https://doi. org/10.12957/reuerj.2019.45584.

19. Minayo MCS. Sampling and saturation in qualitative research: consensuses and controversies. Revista Pesquisa Qualitativa. 2017;5(7):1-12.

20. Minayo MCS. O desafio do conhecimento: pesquisa qualitativa em saúde. 13 $3^{\mathrm{a}}$ ed. São Paulo: Hucitec; 2013.

21. Siqueira CL, Bernadeli ACF, Gasparino RC, Feldman LB, Cunha ICKO, Oliveira RA. Conhecimento de enfermeiros responsáveis técnicos sobre competências gerenciais: um estudo qualitativo. Rev Bras Enferm. 2019 jan/fev;72(1):43-8. http://dx.doi.org/10.1590/0034-7167-2017-0761. PMid:30916266.

22. Ahlin C, Klang-Soderkvist B, Johansson E, Bjorkholm M, Lofmark A. Assessing nursing students' knowledge and skills in performing venepuncture and inserting peripheral venous catheters. Nurse Educ Pract. 2017;23(4):8-14. http://dx.doi.org/10.1016/j.nepr.2017.01.003. PMid:28171853

23. Cant RP, Cooper SJ, Lam L.L. Hospital Nurses' simulation-based education regarding patient safety: a scoping review. Clin Simul Nurs. 2020;28(1):4-8. https://doi.org/10.1016/j.ecns.2019.11.006

24. Teles MG, Mendes-Castillo AMC, Oliveira-Kumakura ARS, Silva JLG. Simulação clínica no ensino de Enfermagem pediátrica: percepção de estudantes. Rev Bras Enferm. 2020 mar 30;73(2):e20180720. http:// dx.doi.org/10.1590/0034-7167-2018-0720. PMid:32236374.

25. Ravik M, Havnes A, Bjork IT. Defining and comparing learning actions in two simulation modalities: students training on a latex arm and each other's arms. J Clin Nurs. 2017;26(23-24):4255-66. http://dx.doi. org/10.1111/jocn.13748. PMid:28152220.

26. Freire P. Pedagogia do oprimido. 50a ed. São Paulo: Paz e Terra; 2014.

27. Oliveira SN, Prado ML, Kempfer SS, Martini JG. Experiential learning in nursing consultation education via clinical simulation with actors: action research. Nurse Educ Today. 2015;35(2):e50-4. http://dx.doi. org/10.1016/j.nedt.2014.12.016.

28. Boostel R, Felix JVC, Bortolato-Major C, Pedrolo E, Vayego SA, Mantovani MF. Stress of nursing students in clinical simulation: a randomized clinical trial. Rev Bras Enferm. 2018;71(3):967-74. http:// dx.doi.org/10.1590/0034-7167-2017-0187. PMid:29924167.

29. Fernandes MTC, Alves CN. Simulação como metodologia na formação de discentes em enfermagem no estágio final da graduação. Atas de Ciências da Saúde. 2019 jan/dez; [citado 2020 abr 22];7:115-25. Disponivel em: https:// pdfs.semanticscholar.org/9f04/1295c2f2809dc60972f5b7c26d4430acc462. pdf

30. Peixoto TASM, Peixoto NMSM. Pensamento crítico dos estudantes de enfermagem em ensino clínico: uma revisão integrativa. Rev Enf Ref. 2017 jun 30;IV Série(13):125-38. http://dx.doi.org/10.12707/RIV16029.

31. Marques LMNSR. As metodologias ativas como estratégias para desenvolver a educação em valores na graduação em enfermagem. Esc Anna Nery. 2018;22(3):e20180023. http://dx.doi.org/10.1590/21779465-ean-2018-0023.

32. Oliveira SN, Sanes MS, Martini JG. Simulação clínica como método de ensino. In: Schneider DG, Ramos FRS. Ensino simulado e deliberação moral: contribuições para a formação profissional em saúde. Porto Alegre: Moriá; 2019.

33. Salgueiro OAS, Bastos ML, Braga LM, Arreguy-Sena C, Melo MN Parreira PMSD. Práticas de enfermagem no cateterismo venoso periférico: a flebite e a segurança do paciente doente. Texto Contexto Enferm. 2019;28:e20180109. http://dx.doi.org/10.1590/1980-265XTCE-2018-0109.

34. Meska MHG, Mazzo A, Jorge BM, Souza-Junior VD, Negri EC, Chayamiti EMPC. Retenção urinária: implicações do treino simulado de baixa fidelidade na autoconfiança do enfermeiro. Rev Esc Enferm USP. 2016 set/ out;50(5):831-7. http://dx.doi.org/10.1590/s0080-623420160000600017. PMid:27982403.

35. Hernández-Padilla JM, Granero-Molina J, Márquez-Hernández VV, Cortés-Rodríguez AE, Fernández-Sola C. Efeitos de um workshop de simulação sobre a competência em punção arterial de estudantes de enfermagem. Acta Paul Enferm. 2016;29(6):678-85. http://dx.doi. org/10.1590/1982-0194201600095.

36. Costa R, Medeiros SM, Martins J, Coutinho V. Simulation in training nurses: reflections and justifications based on bioethics and human rights approaches. Acta Bioeth. 2018;24(1):31-8.

37. Oliveira SN, Massaroli A, Martini JG, Rodrigues J. Da teoria à prática, operacionalizando a simulação clínica no ensino de Enfermagem. Rev Bras Enferm. 2018;71(Suppl 4):1791-8. http://dx.doi.org/10.1590/00347167-2017-0180. PMid:30088655.

38. Coelho A, Parola V, Cardoso D, Duarte S, Almeida M, Apóstolo J. O uso do simulador de velhice em estudantes de enfermagem: uma scoping review. Rev Enf Ref. 2017;serIV(14):147-58. http://dx.doi.org/10.12707/ RIV17050.

39. Bellaguarda MLR, Knihs NS, Canever BP, Tholl AD, Alvarez AG, Teixeira GC. Simulação realística como ferramenta de ensino na comunicação de situação crítica em cuidados paliativos. Esc Anna Nery. 2020;24(3):e20190271. http://dx.doi.org/10.1590/2177-9465ean-2019-0271 